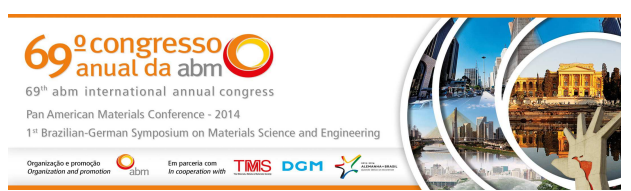

Tema: Fundição

\title{
VERIFICAÇÃO ESTATÍSTICA DA ANÁLISE DE INCLUSÕES DE ESCÓRIA EM AMOSTRAS FERROSAS COLETADAS NO ENTORNO DO MORRO DE ARAÇOIABA*
}

\section{Resumo}

\author{
Rafael Rocha Maia \\ Marcelli Susaki ${ }^{2}$ \\ Cesar Roberto de Farias Azevedo ${ }^{3}$ \\ Fernando José Gomes Landgraf
}

No presente trabalho, analisaram-se três amostras metálicas ferrosas provenientes da Real Fábrica de Ferro de Ipanema (RFFI) outras três do sitio arqueológico de Sardinha (SAS) em busca de uma assinatura química dos processos produtivos destes objetos com base num modelo de regressão linear dos resultados de análise química de inclusões não metálicas proposto por Dillmann et al. [1]. Foram usadas técnicas de caracterização microestrutural associadas com microanálise EDS (Energy dispersive $X$-ray spectroscopy) destas inclusões. Com os resultados de microanálise, foram construídos gráficos de relações entre os principais constituintes destas inclusões (\%FeO versus $\% \mathrm{SiO}_{2}, \% \mathrm{SiO}_{2}$ versus $\% \mathrm{Al}_{2} \mathrm{O}_{3}$, $\% \mathrm{Al}_{2} \mathrm{O}_{3}$ versus $\% \mathrm{MgO}$ e $\% \mathrm{CaO}$ versus $\% \mathrm{~K}_{2} \mathrm{O}$ ). Utilizou-se, então, a técnica de regressão linear (com reta passando e sem passar pela origem) para cada uma destas relações de modo a obter e avaliar o valor do coeficiente de determinação linear $R^{2}$. Aplicou-se, ainda, 0 teste de t de Student para avaliar a hipótese de "reta passando forçosamente pela origem", como proposto por Dillmann et al. [1]. Não foi possível obter a assinatura química dos processos produtivos, visto que a análise estatística dos dados mostrou uma incoerência no modelo utilizado por Dillmann et al. [1], que assumia que a regressão linear passava pela origem dos eixos.

Palavras-chave: Arqueometalurgia; Microanálise de inclusões; Análise estatística; Processos de produção.

\section{VERIFICATION OF STATISTICAL ANALYSIS OF SLAG INCLUSIONS IN SAMPLES FROM FERROUS COLLECTED IN THE VICINITY OF MORRO ARAÇOIABA}

\begin{abstract}
In the present study, analyzed three samples from ferrous metal Real Iron Factory Ipanema (RIFI) three other archaeological Site in Sardinia (ASS) in search of a chemical signature of the production processes of these objects based on a linear regression model the results of chemical analysis of non-metallic inclusions proposed by Dillmann et al. [1]. Microstructural characterization techniques used were associated with EDS microanalysis (Energy dispersive Xray spectroscopy) of these inclusions. With the results of microanalysis, graphs were constructed relationships between the major components of these inclusions (\% $\mathrm{FeO}$ versus $\mathrm{SiO} 2 \%$, versus $\mathrm{SiO} 2 \% \mathrm{Al} 2 \mathrm{O} 3 \%, \% \% \mathrm{Al} 2 \mathrm{O} 3$ versus $\mathrm{MgO}$ and $\mathrm{CaO} \%$ versus \% $\mathrm{K} 2 \mathrm{O}$ ). Then used the technique of linear regression (with line through and without passing through the origin) for each of these relationships to obtain and evaluate the value of the linear coefficient of determination $R^{2}$. Applied also the Student $t$ test to evaluate the hypothesis "line passing through the origin forcibly," as proposed by Dillmann et al. [1]. Unable to get the chemical signature of the productive processes, as the statistical analysis of the data showed an inconsistency in the model used by Dillmann et al. [1], which assumed that the linear regression passing through the origin of the axes.
\end{abstract}

Keywords: Archaeometallurgy; Microanalysis of inclusions; Statistical analysis; Production processes.

1 Mestrando em Engenharia de Metalúrgica e de Materiais, Depto. Eng. Metalúrgica e de Materiais, Escola Politécnica, Universidade São Paulo (EPUSP), São Paulo. SP, Brasil.

2 Graduando, Eng. de Metalúrgica e de Materiais, Depto. Eng. Metalúrgica e de Materiais, EPUSP, São Paulo, SP, Brasil.

3 Professor, Depto. de Engenharia Metalúrgica e de Materiais, EPUSP, São Paulo, SP, Brasil.

4 Professor, Depto. de Engenharia Metalúrgica e de Materiais, EPUSP, Presidente do Instituto de Pesquisa Tecnológica do Estado de São Paulo (IPT), cidade, estado, Brasil.

\footnotetext{
* Contribuição técnica ao $69^{\circ}$ Congresso Anual da ABM - Internacional e ao 14ํㅡㄹ ENEMET - Encontro Nacional de Estudantes de Engenharia Metalúrgica, de Materiais e de Minas, 21 a 25 de julho de 2014, São Paulo, SP, Brasil.
} 


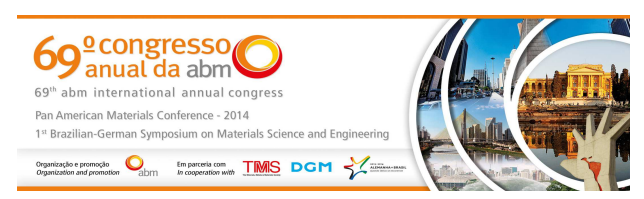

\section{INTRODUÇÃO}

A Real Fábrica de Ferro de São João de Ipanema é um importante episódio da história do Brasil (século XIX) e refere-se à instalação de altos-fornos às margens do Rio Ipanema, no pé do Morro de Araçoiaba, próximo à Sorocaba [2-5]. Este evento deu continuidade a três episódios anteriores de instalação de operações de redução de minério de ferro por redução direta e forjamento (processo de redução direta do minério de ferro), em outra localização no mesmo Morro, uma deles denominado como Sítio de Sardinha (século XVI) [2-5]. Durante o período que antecede o século XIX, uma parte das operações metalúrgicas referentes à obtenção de ferro realizava-se abaixo da temperatura de fusão do metal (processo de redução direta), até a implantação do processo de redução indireta de Ipanema, que consistia da redução do óxido de ferro em ferro gusa e depois do refino do ferro gusa, rico em carbono, em ferro. Ambos os processos produziam artefatos metálicos com uma grande presença de partículas não metálicas na microestrutura do material $[1,5,7]$.

Estas partículas microscópicas, chamadas de inclusões de escória (IE), contêm vários tipos de óxidos, que não foram reduzidos ou que foram formados pela "reoxidação" de elementos durante o processo produtivo [1,5,7]. A composição química das diversas inclusões de escória (IE) encontradas na microestrutura de um artefato arqueológico ferroso deve conter importantes informações sobre a composição das diferentes matérias primas e insumos (minérios, cinzas de carvão, tijolos refratários, revestimentos de paredes dos fornos, fluxantes, etc.) usados no seu sistema produtivo. Além disto, a composição química das IE também podem revelar algumas informações sobre o processo de fabricação do ferro, já que a constituição química e microestrutura da escória também dependem das condições termodinâmicas e cinéticas do sistema produtivo [1,5,7-11].

Por exemplo, em 1998 Buchwald et al. [8] analisaram as inclusões de escória de artefatos ferrosos provenientes de diferentes sítios. Eles notaram que as inclusões de objetos medievais de ferro provenientes da Dinamarca tinham uma relação entre os teores médios de $\% \mathrm{SiO}_{2}$ e de $\% \mathrm{Al}_{2} \mathrm{O}_{3}$ diferente das inclusões de objetos provenientes da Noruega e Suécia. Este estudo aventou a possibilidade de identificar a origem de alguns artefatos ferrosos pela análise de inclusão das escória com foco na relação entre os teores médios de $\% \mathrm{SiO}_{2}$ e de $\% \mathrm{Al}_{2} \mathrm{O}_{3}$.

Em 2007, Dillmann et al. [1] analisaram as inclusões de escória de artefatos ferrosos provenientes de diversas construções medievais francesas. Eles notaram, usando microanálise EDS que os teores variavam entre inclusões do mesmo artefato. Usando técnicas básicas de regressão linear, notaram que a razão entre os teores de certos óxidos presentes nas inclusões mantinham proporções similares, apesar da variabilidade dos resultados de microanálise química nas inclusões de escória de uma mesma amostra. Para tanto, eles usaram os resultados de microanálise química das inclusões de escória e criaram gráficos relacionando os diversos teores de óxidos $\left(\mathrm{SiO}_{2}, \mathrm{CaO}, \mathrm{K}_{2} \mathrm{O}, \mathrm{Al}_{2} \mathrm{O}_{3}\right.$ e $\left.\mathrm{MgO}\right)$ nas inclusões dos diversos artefatos de mesma origem. Eles usaram técnicas de regressão linear (com reta passando pela origem e descarte de resultados atpe que o valor fosse obtido $R^{2}>0,7$ ) para estabelecer o valor da razão entre estes diversos óxidos para cada um dos sítios arqueológico investigados. Eles mostraram com este método que seria possível determinar a assinatura do "processo produtivo de cada um dos sítios arqueológicos".

Em 2009, Blakelock et al. [7] tentaram reproduzir experimentalmente diferentes processos de fabricação, como redução, refino e forjamento. Eles compararam os

* Contribuição técnica ao $69^{\circ}$ Congresso Anual da ABM - Internacional e ao 14ํㅡㄹ ENEMET - Encontro Nacional de Estudantes de Engenharia Metalúrgica, de Materiais e de Minas, 21 a 25 de julho de 2014, São Paulo, SP, Brasil. 

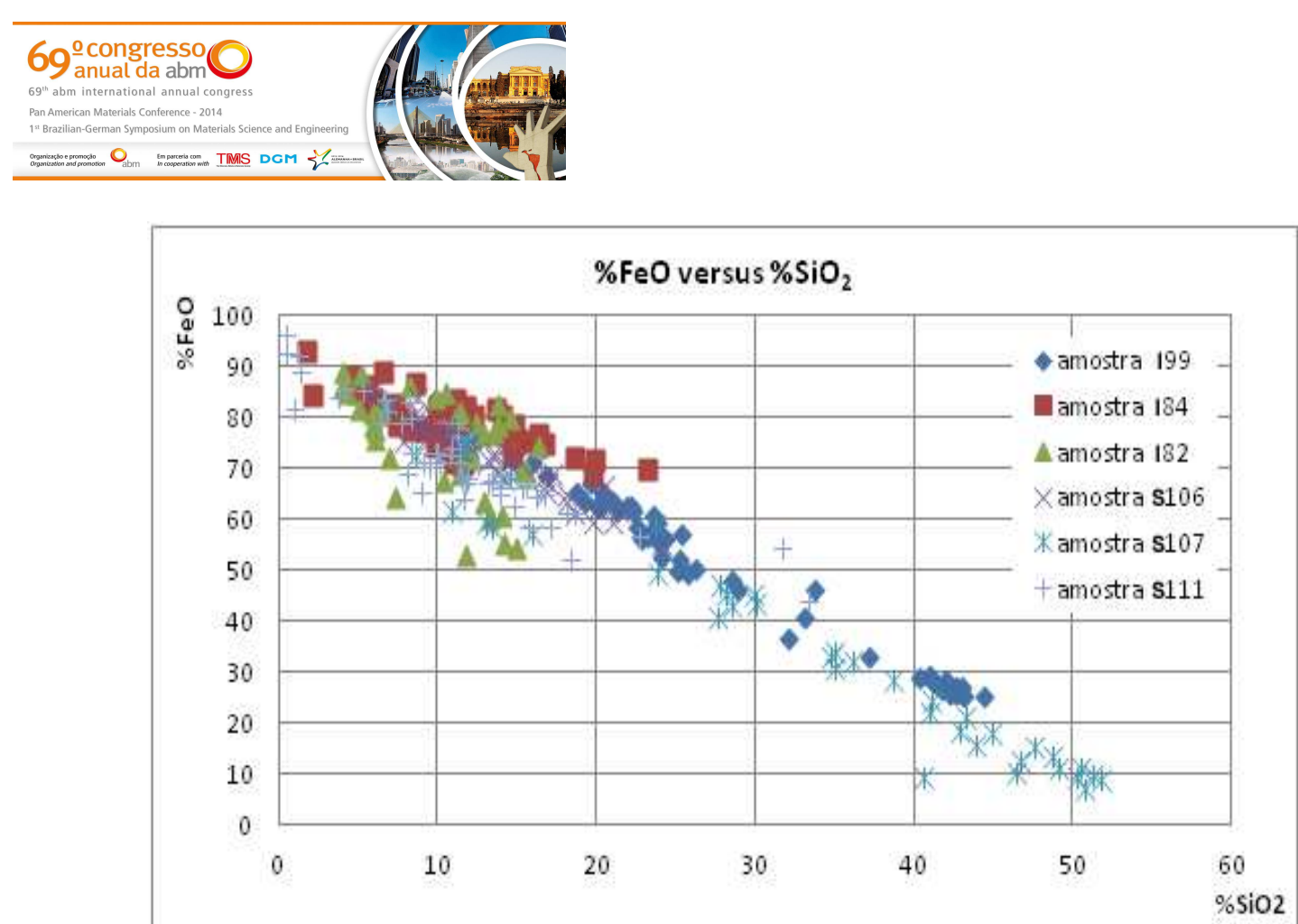

Figura 1. Teores de $\mathrm{FeO}$ e $\mathrm{SiO}_{2}$ nas inclusões de escória. A Figuras 2 a 4 representam as diversas relações de teores de óxidos estudadas para os seis artefatos $\left(\mathrm{SiO}_{2} \times \mathrm{Al}_{2} \mathrm{O}_{3} ; \mathrm{Al}_{2} \mathrm{O}_{3} \times \mathrm{MgO}\right.$; e $\mathrm{CaO} \times$ $\mathrm{K}_{2} \mathrm{O}$ ).

Grosseiramente é possível estabelecer uma relação direta entre estes teores de óxidos. Adicionalmente as amostras do Sítio do Sardinha apresentam-se deslocadas para o quadrante superior direito para todas as figuras.

Foi adotado um modelo de regressão linear para os resultados obtidos para cada relação estudada, cujo comportamento pode ser expresso pela equação do tipo $y=\alpha x+b$, onde $x$ e y são as variáveis da função, $\alpha$ é a inclinação da reta e $b$ é o intercepto onde a reta corta o eixo da variável y. A Tabela 1 apresenta os resultados dos testes de significância para os interceptos das regressões lineares dos resultados de microanálise química das inclusões de escória da Fábrica de Ipanema, enquanto a tabela 2 traz os os resultados dos artefatos do Sítio de Sardinha. Ambas as análises se concentram nas relações mostradas nas Figuras de 2 a 4 . Para realizar o teste de significância do intercepto aplicou-se o teste t de Student, onde é calculado o valor $|t|$. Caso este valor seja maior que dois $(|t|>2)$, o intercepto é significativo e a regressão linear não pode passar pela origem [10]. Em outro teste de significância do intercepto, calcula-se o valor da probabilidade de significância $p$ (para 95\%) [12]. Neste caso um valor de $p$ menor do que 0,05 significa que o valor do intercepto é representativo e que, portanto, a regressão linear não poderá passar pela origem. Observa-se de um modo genérico que $78 \%$ dos coeficientes de determinação linear $\left(R^{2}\right)$ estão abaixo de 0,7 e que $61 \%$ das regressões lineares (só para as amostras, conjuntos não foram considerados) não passam pela origem. Estes números serão tratados com mais detalhe na discussão a seguir.

\footnotetext{
* Contribuição técnica ao $69^{\circ}$ Congresso Anual da ABM - Internacional e ao 14ํㅡㄹ ENEMET - Encontro Nacional de Estudantes de Engenharia Metalúrgica, de Materiais e de Minas, 21 a 25 de julho de 2014, São Paulo, SP, Brasil.
} 


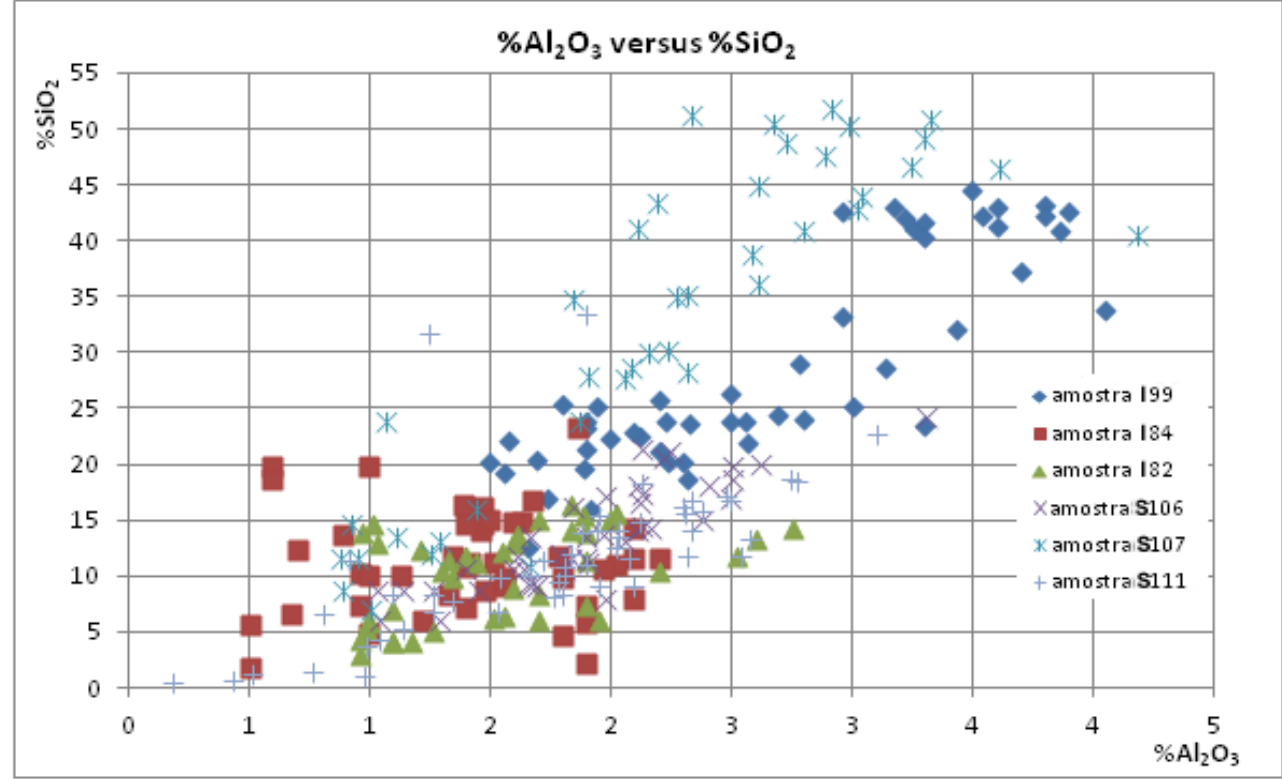

Figura 2. Teores $\mathrm{SiO}_{2}$ e $\mathrm{Al}_{2} \mathrm{O}_{3}$ nas inclusões de escória.

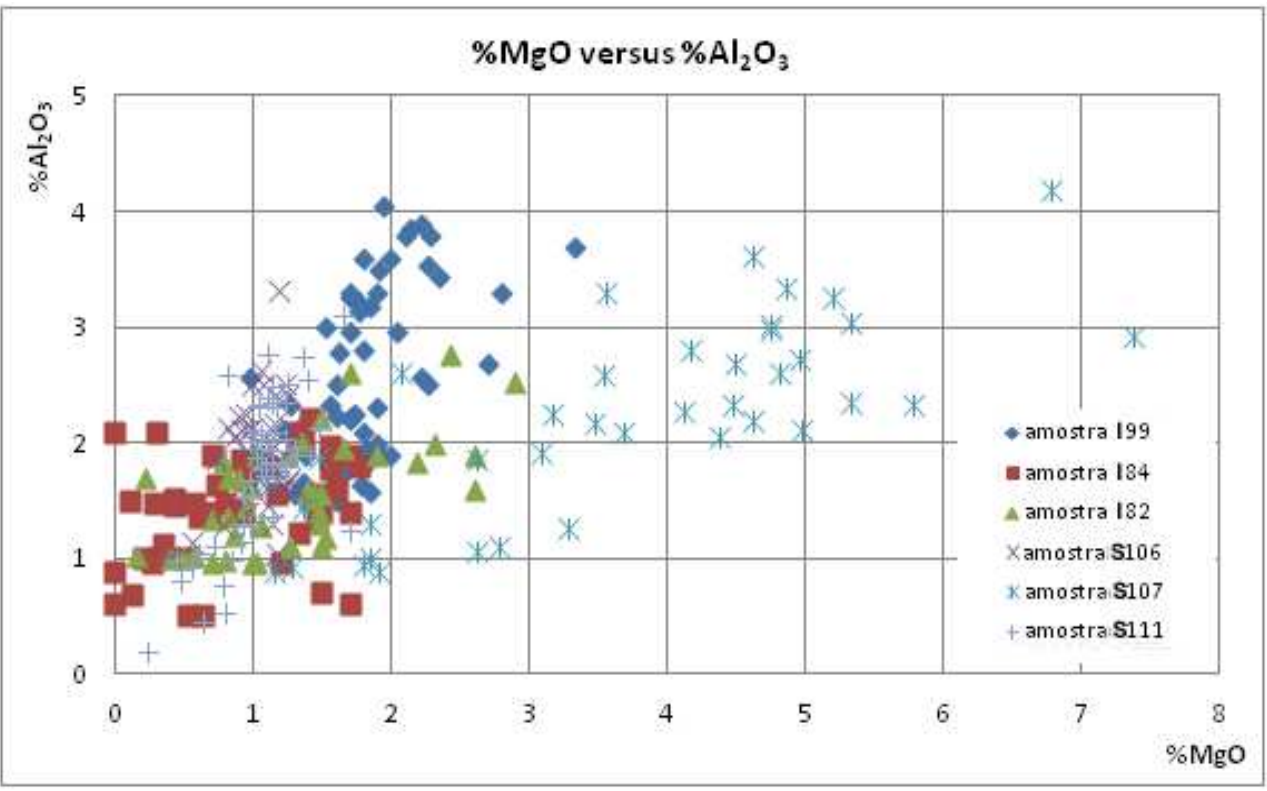

Figura 3. Teores de $\mathrm{MgO}$ e $\mathrm{Al}_{2} \mathrm{O}_{3}$ encontrados nas inclusões de escória. 


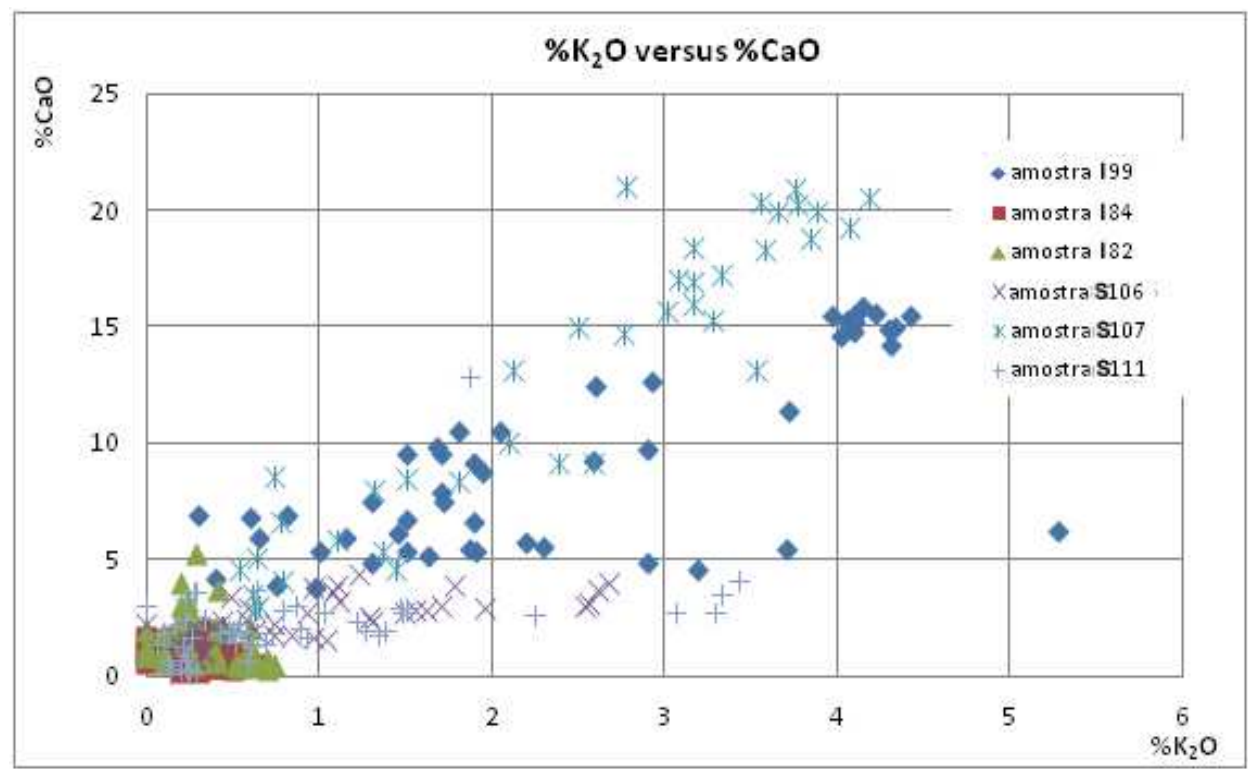

Figura 4. Teores de $\mathrm{CaO}$ e $\mathrm{K}_{2} \mathrm{O}$ encontrados nas inclusões de escória.

Tabela 1. Amostras do Sítio de Ipanema. Teste de significância do intercepto e análise de regressão linear $\mathrm{y}=\mathrm{ax}+\mathrm{b}$ para as relações $\% \mathrm{SiO}_{2}$ versus $\% \mathrm{Al}_{2} \mathrm{O}_{3}, \% \mathrm{Al}_{2} \mathrm{O}_{3}$ versus $\% \mathrm{MgO}$ e $\% \mathrm{CaO}$ versus $\% \mathrm{~K}_{2} \mathrm{O}$.

\begin{tabular}{|c|c|c|c|c|c|c|c|}
\hline Relação & Amostra & $|t|$ & $p$ & $\begin{array}{l}\text { Pode passar } \\
\text { pela Origem? }\end{array}$ & $\begin{array}{l}\text { Inclinação } \\
\text { (a) }\end{array}$ & $\begin{array}{l}\text { Intercepto } \\
\text { (b) }\end{array}$ & $\mathrm{R}^{2}$ \\
\hline \multirow{4}{*}{$\begin{array}{l}\% \mathrm{SiO}_{2} \text { versus } \\
\% \mathrm{Al}_{2} \mathrm{O}_{3}\end{array}$} & 99 & 0,0 & 0,97 & SIM & 10,8 & -0.1 & 0,74 \\
\hline & 82 & 1,9 & 0,07 & SIM & 7,2 & 11,0 & 0,24 \\
\hline & 84 & 4,8 & 0,00 & NÃO & 4,2 & 3,6 & 0,32 \\
\hline & Conjunto & 3,0 & 0,00 & $N \tilde{A O}$ & 11,1 & 3,8 & 0,73 \\
\hline \multirow{4}{*}{$\begin{array}{l}\% \mathrm{Al}_{2} \mathrm{O}_{3} \text { versus } \\
\% \mathrm{MgO}\end{array}$} & 99 & 2,3 & 0,03 & NÃO & 0,9 & 1,0 & 0,32 \\
\hline & 82 & 7,4 & 0,00 & NÄO & 0,3 & 1,2 & 0,13 \\
\hline & 84 & 9,4 & 0,00 & NÃO & 0,5 & 1,0 & 0,41 \\
\hline & Conjunto & 0,9 & 0,00 & $N \tilde{O} O$ & 0,8 & 0,8 & 0,44 \\
\hline \multirow{4}{*}{$\begin{array}{l}\% \mathrm{CaO} \text { versus } \\
\% \mathrm{~K}_{2} \mathrm{O}\end{array}$} & 99 & 4,5 & 0,00 & $\mathrm{NÃO}$ & 0,4 & 1,6 & 0,62 \\
\hline & 82 & 5,9 & 0,00 & NÃO & $-0,9$ & 1,1 & 0,03 \\
\hline & 84 & 6,6 & 0,00 & NÃO & $-1,0$ & 1,8 & 0,06 \\
\hline & Conjunto & 0,3 & 0,00 & $N A \tilde{O}$ & 3,2 & 0,7 & 0,83 \\
\hline
\end{tabular}

${ }^{*} R^{2}$ é calculada para a reta sem impor passar pela origem

Tabela 2 . Amostras do Sítio de Sardinha. Teste de significância do intercepto e análise de regressão linear $\mathrm{y}=\mathrm{ax}+\mathrm{b}$ para as relações $\% \mathrm{SiO}_{2}$ versus $\% \mathrm{Al}_{2} \mathrm{O}_{3}, \% \mathrm{Al}_{2} \mathrm{O}_{3}$ versus $\% \mathrm{MgO}$ e $\% \mathrm{CaO}$ versus $\% \mathrm{~K}_{2} \mathrm{O}$.

\begin{tabular}{|c|c|c|c|c|c|c|c|}
\hline Relação & Amostra & $|t|$ & $p$ & $\begin{array}{l}\text { Pode passar } \\
\text { pela Origem? }\end{array}$ & $\begin{array}{l}\text { Inclinação } \\
\text { (a) }\end{array}$ & $\begin{array}{l}\text { Intercepto } \\
\text { (b) }\end{array}$ & $\mathrm{R}^{2}$ \\
\hline \multirow{4}{*}{$\begin{array}{l}\% \mathrm{SiO}_{2} \text { versus } \\
\% \mathrm{Al}_{2} \mathrm{O}_{3}\end{array}$} & 106 & 1.7 & 0.10 & SIM & 8.5 & -2.7 & 0,72 \\
\hline & 107 & 0.3 & 0.80 & SIM & 15.1 & -0.8 & 0,83 \\
\hline & 111 & 0.1 & 0.92 & SIM & 6.7 & -0.2 & 0,42 \\
\hline & Conjunto & 3.4 & 0.00 & NÃO & 13.8 & -8.3 & 0,54 \\
\hline \multirow{4}{*}{$\begin{array}{l}\% \mathrm{Al}_{2} \mathrm{O}_{3} \text { versus } \\
\% \mathrm{MgO}\end{array}$} & 106 & 2.9 & 0.01 & NÃO & 0.8 & 1.1 & 0,13 \\
\hline & 107 & 4.1 & 0.00 & NÃO & 0.5 & 0.4 & 0,92 \\
\hline & 111 & 0.4 & 0.67 & SIM & 1.6 & 0.1 & 0,21 \\
\hline & Conjunto & 19.1 & 0.00 & NÃO & 0.3 & 1.4 & 0,43 \\
\hline \multirow{4}{*}{$\begin{array}{l}\% \mathrm{CaO} \text { versus } \\
\% \mathrm{~K}_{2} \mathrm{O}\end{array}$} & 106 & 12.2 & 0.00 & NÃO & 0.3 & 2.2 & 0,24 \\
\hline & 107 & 1.8 & 0.08 & SIM & 4.4 & 2.7 & 0,62 \\
\hline & 111 & 4.5 & 0.00 & NÃO & 0.9 & 1.5 & 0,23 \\
\hline & Conjunto & 1.1 & 0.27 & SIM & 3.5 & 0.6 & 0,54 \\
\hline
\end{tabular}

${ }^{*} R^{2}$ é calculada para a reta sem impor passar pela origem

\footnotetext{
* Contribuição técnica ao $69^{\circ}$ Congresso Anual da ABM - Internacional e ao 14ํㅡㄹ ENEMET - Encontro Nacional de Estudantes de Engenharia Metalúrgica, de Materiais e de Minas, 21 a 25 de julho de 2014, São Paulo, SP, Brasil.
} 


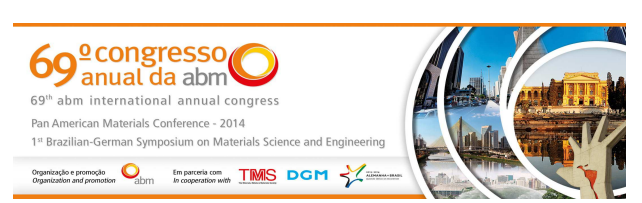

\subsection{Discussão}

A Figura 1 apresenta uma anticorrelação, ou relação inversa, entre os teores de $\mathrm{FeO}$ e $\mathrm{SiO}_{2}$ observados para as amostras do Sardinha e de Ipanema, distribuindo-se linearmente numa faixa que vai de 20 a $90 \%$ de $\mathrm{FeO}$, cuja extrapolação tende a $100 \%$ de $\mathrm{FeO}$. Isto sugere que o $\mathrm{FeO}$ é um elemento diluidor da composição química das inclusões. Dadas as condições oxidantes das etapas finais do processo de fabricação e a diferentes distâncias de difusão do oxigênio até cada uma das inclusões, é razoável imaginar que as inclusões tem uma única composição básica (excluindo o $\mathrm{FeO}$ ) e os diferentes teores de $\mathrm{FeO}$ em cada uma levam ao comportamento exibido. Assim sendo, as proporções entre os outros óxidos seriam constantes, resultando em retas nas relações entre eles.

As Figuras 2 a 4 e os resultados das Tabelas 1 e 2 deveriam permitir confirmar essa hipótese, mas a análise do resultados mostra-se mais complexa. No caso das amostras aqui analisadas, existe a dúvida se elas foram produzidos por redução direta ou indireta e, mais ousadamente, se é possível diferenciar processos de fabricação pela análise das inclusões, seguindo metodologia proposta por Dillmann et al. [1].

Dentre as questões que esse conjunto de hipóteses enseja, algumas exigem um tratamento estatístico. Portanto é possível os realizar seguintes questionamentos:

- $\quad$ Os resultados experimentais das Figuras 2 a 4 podem ser ajustados por uma reta?

- Quais os coeficientes de correlação linear $\left(R^{2}\right)$ encontrados para cada uma das relações estudadas (para cada amostra e para o mesmo conjunto de amostras)?

- É razoável supor que os resultados possam ser ajustados por uma reta que passe por zero?

Os resultados mostrados nas Tabelas 1 e 2 usando dois métodos distintos para investigar a significância do intercepto indicaram que apenas cinco das vinte e quatro regressões lineares estudadas podem passar pela origem $\left(\% \mathrm{SiO}_{2}\right.$ versus $\% \mathrm{Al}_{2} \mathrm{O}_{3}$ para o objeto 199 e $\% \mathrm{Al}_{2} \mathrm{O}_{3}$ versus $\% \mathrm{MgO}$ também para o objeto 199 e $\% \mathrm{SiO}_{2}$ versus $\% \mathrm{Al}_{2} \mathrm{O}_{3}$ para as amostra $\mathrm{S} 106$ e S107). Todas as demais dezenove regressões não podem passar pela origem, contrariando a metodologia proposta anteriormente [1].

Adicionalmente, os valores do coeficiente de determinação $\left(R^{2}\right)$ da regressão simples (vide Tabelas 1 e 2) foram comparados aos valores da regressão linear passando pela origem. Considerando-se os valores de $\mathrm{R}^{2}$ de cada regressão linear simples de cada artefato mostrados na Tabelas 1 e 2, pode-se afirmar que $22 \%$ das amostras apresentam valores de $R^{2}$ próximos do valor mínimo proposto anteriormente $\left(\mathrm{R}^{2}>0,7\right)$ (relação $\% \mathrm{SiO}_{2}$ versus $\% \mathrm{Al}_{2} \mathrm{O}_{3}$ para a amostra 199 , relação $\% \mathrm{SiO}_{2}$ versus $\% \mathrm{Al}_{2} \mathrm{O}_{3}$ para as amostras $\mathrm{S} 106$ e $\mathrm{S} 107$, e relação $\% \mathrm{Al}_{2} \mathrm{O}_{3}$ versus $\% \mathrm{MgO}$ para a amostra S107), indicando a inexistência de uma regressão linear entre as variáveis estudadas, contrariando a metodologia proposta anteriormente [1]. Quando se compara os resultados de regressão linear obtidos para cada amostra e para o conjunto de amostras do mesmo sítio (vide Tabelas 1 e 2) é possível observar uma diferença significativa. As análises do conjunto de amostras da Fábrica de Ipanema apresentaram valores de $R^{2}$ maiores que 0,7 para as relações $\mathrm{Al}_{2} \mathrm{O}_{3}$ versus $\mathrm{SiO}_{2}$ e $\mathrm{CaO}$ versus $\mathrm{K}_{2} \mathrm{O}$, enquanto que as análises do conjunto de amostras do sítio Sardinha não apresentaram nenhum valor de $R^{2}>0,7$. No entanto, quando se observam os resultados de valores individuais do Sítio de Sardinha (vide

\footnotetext{
* Contribuição técnica ao $69^{\circ}$ Congresso Anual da ABM - Internacional e ao 14ํㅡㄹ ENEMET - Encontro Nacional de Estudantes de Engenharia Metalúrgica, de Materiais e de Minas, 21 a 25 de julho de 2014, São Paulo, SP, Brasil.
} 


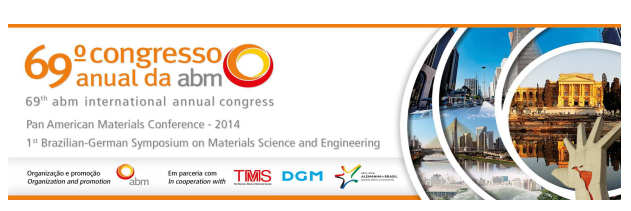

Tabela 2), em 33\% das análises individuais apresentam $R^{2}>0,7$, enquanto que para Ipanema (tabela 1) apenas $11 \%$ das análises individuais apresentam $R^{2}>0,7$. Alguns destes resultados, para os teores de $\mathrm{MgO}$ versus $\mathrm{Al}_{2} \mathrm{O}_{3}$, são mostrados nas Figuras 5-a e 5-b. Esta análise permite questionar a eficácia da técnica de se usar a análise do conjunto de resultados para a obtenção da assinatura do processo produtivo de Ipanema e do sítio Sardinha, principalmente se for incluída a incerteza quanto à datação de cada um dos artefatos analisados do ponto de vista arqueológico.

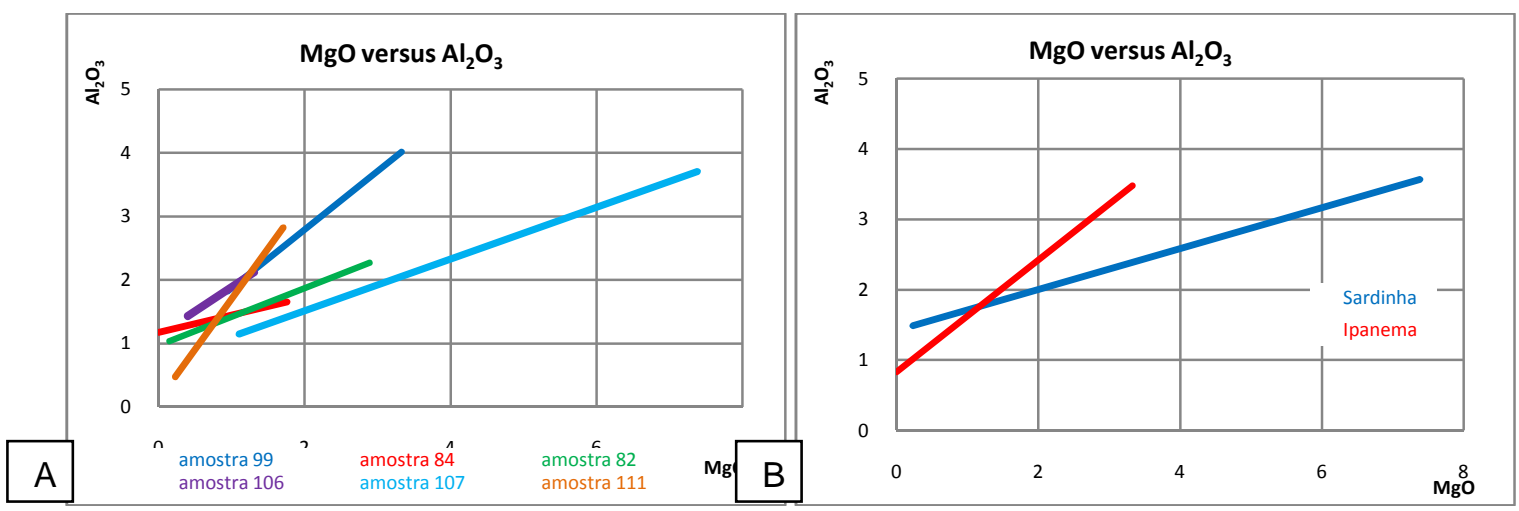

Figura 5. Teores de $\mathrm{SiO}_{2}$ e $\mathrm{Al}_{2} \mathrm{O}_{3}$ nas inclusões de escória. Tratamento dos dados, usando a regressão linear sem impor passar pela origem: a) amostras individuais; e b) conjunto de dados.

As Figuras 6-a e 6-b ilustram os resultados de regressão linear impondo passagem pela origem dos teores de $\mathrm{MgO}$ versus $\mathrm{Al}_{2} \mathrm{O}_{3}$, obtidos segundo metodologia proposta por Dillmann et al. [1].
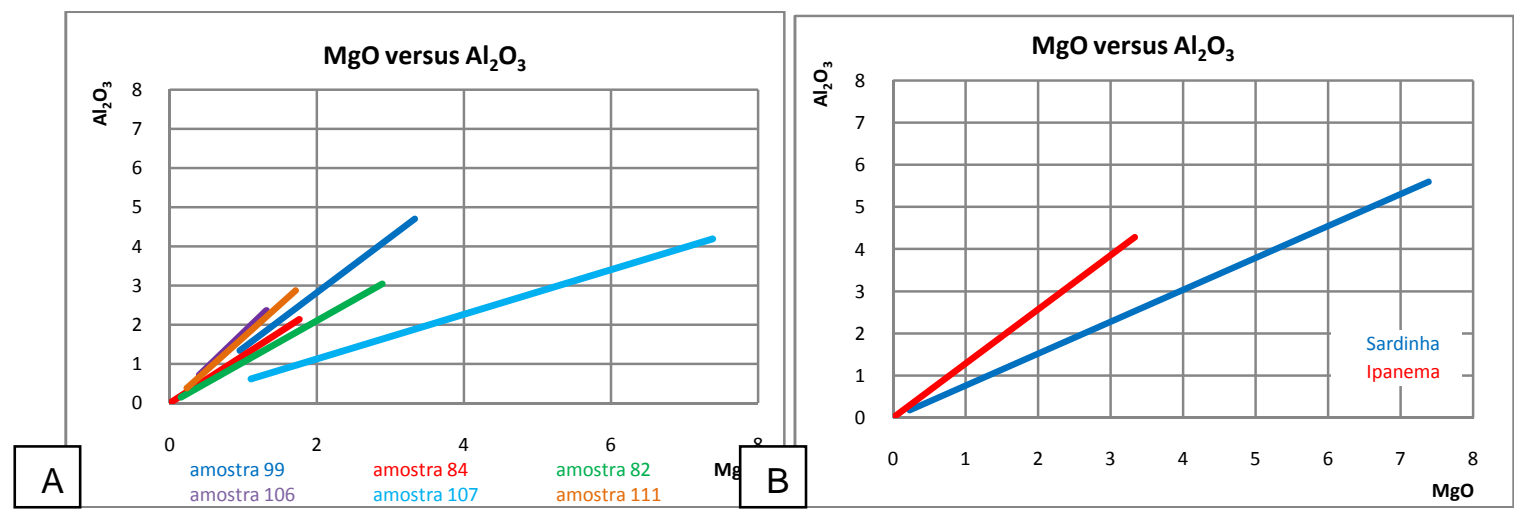

Figura 6. Teores de $\mathrm{SiO}_{2}$ e $\mathrm{Al}_{2} \mathrm{O}_{3}$ nas inclusões de escória. Tratamento dos dados, usando a regressão linear impondo passagem pela origem: a) amostras individuais; e b) conjunto de dados.

Podemos então comparar duas situações de regressão linear: regressão impondo passagem pela origem (Figuras 6-a e 6-b) versus regressão linear simples (Figuras 5-a e 5-b). Percebe-se claramente que a hipótese da regressão linear impondo passagem pela origem, além de ser incorreta para o presente conjunto de dados, aumenta de forma artificial os valores de $R^{2}$ (vide Tabela 3 ).

\footnotetext{
* Contribuição técnica ao $69^{\circ}$ Congresso Anual da ABM - Internacional e ao 14ํㅡㄹ ENEMET - Encontro Nacional de Estudantes de Engenharia Metalúrgica, de Materiais e de Minas, 21 a 25 de julho de 2014, São Paulo, SP, Brasil.
} 


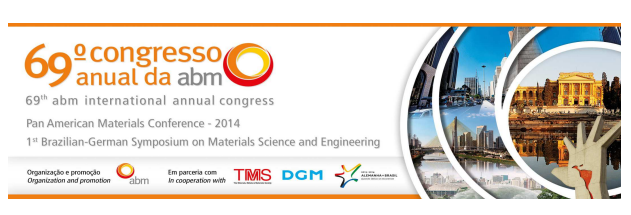

Tabela 3. Valores dos coeficientes de correlação linear $\left(R^{2}\right)$ comparando regressão linear passando forçosamente pela origem e sem forçar.

\begin{tabular}{|c|c|c|c|c|c|c|}
\hline \multirow[b]{2}{*}{ Amostra } & \multicolumn{3}{|c|}{ Reta passando pela origem } & \multicolumn{3}{|c|}{ Sem forçar a reta a passar pela origem } \\
\hline & $\begin{array}{c}\% \mathrm{Al}_{2} \mathrm{O}_{3} \mathrm{x} \\
\% \mathrm{MgO}\end{array}$ & $\begin{array}{r}\% \mathrm{CaO} \\
\mathrm{x} \% \mathrm{~K}_{2} \mathrm{O}\end{array}$ & $\begin{array}{c}\% \mathrm{SiO}_{2} \mathbf{x} \\
\% \mathrm{Al}_{2} \mathrm{O}_{3}\end{array}$ & $\begin{array}{c}\% \mathrm{Al}_{2} \mathrm{O}_{3} \mathrm{x} \\
\% \mathrm{MgO}\end{array}$ & $\begin{array}{c}\% \mathrm{CaO} x \\
\% \mathrm{~K}_{2} \mathrm{O}\end{array}$ & $\begin{array}{c}\% \mathrm{SiO}_{2} \times \\
\% \mathrm{Al}_{2} \mathrm{O}_{3}\end{array}$ \\
\hline 199 & 0,94 & 0,92 & 0,74 & 0,32 & 0,62 & 0,74 \\
\hline 182 & 0,74 & 0,48 & 0,77 & 0,13 & 0,03 & 0,24 \\
\hline 184 & 0,88 & 0,33 & 0,88 & 0,41 & 0,06 & 0,32 \\
\hline Conjunto & 0,88 & 0,88 & 0,9 & 0,44 & 0,83 & 0,73 \\
\hline S106 & 0,94 & 0,61 & 0,94 & 0,13 & 0,24 & 0,72 \\
\hline S107 & 0,98 & 0,93 & 0,96 & 0,92 & 0,62 & 0,83 \\
\hline S111 & 0,94 & 0,54 & 0,86 & 0,21 & 0,23 & 0,42 \\
\hline Conjunto & 0,75 & 0,71 & 0,83 & 0,43 & 0,54 & 0,54 \\
\hline
\end{tabular}

- $\quad$ Para cada regressão linear impondo passagem pela origem de cada artefato, pode-se afirmar agora que 14 das 18 relações estudadas apresentam valores de $\mathrm{R}^{2}$ próximos do valor mínimo considerado $\left(R^{2}>0,7\right)$, indicando a existência de uma regressão linear entre as variáveis estudadas.

- Para cada regressão linear impondo passagem pela origem do conjunto de resultados, todas as relações estudadas apresentam valores de $R^{2}$ próximos do valor mínimo considerado por Dillmann et al. [1] $\left(R^{2}>0,7\right)$, indicando a existência de uma regressão linear entre as variáveis estudadas.

Em termos metalúrgicos a discriminação do intercepto, forçando que a regressão passe pela origem, não é justificável já que os elementos presentes no minério são independentes entre si. Logo tanto a análise de significância do intercepto como as considerações metalúrgicas tornam inválida a hipótese proposta por Dillmann et al. [1] de que na análise dos dados, para verificar a existência de uma assinatura de um sítio, a regressão deve passar pela origem.

Estes resultados ilustram que $R^{2}$ deixa de ter qualquer valor significativo para a análise de regressão linear passando pela origem, conforme usado na metodologia proposta anteriormente [1]. Assim sendo não foi possível determinar a assinatura química do sítio arqueológico de Ipanema.

Finalmente, as Figuras 2 a 4 representam as diversas relações de teores de óxidos estudadas para os seis artefatos $\left(\mathrm{SiO}_{2} \times \mathrm{Al}_{2} \mathrm{O}_{3} ; \mathrm{Al}_{2} \mathrm{O}_{3} \times \mathrm{MgO}\right.$; e $\left.\mathrm{CaO} \times \mathrm{K}_{2} \mathrm{O}\right)$. As amostras do Sítio do Sardinha apresentam-se deslocadas para o quadrante superior direito para figuras 2 a 4 . Adicionalmente na anticorrelação da Figura 1 as amostras de Sardinha estão localizadas preferencialmente no quadrante inferior direito. Ambos referem-se ao menos teor de $\mathrm{FeO}$ das amostras do Sítio de Sardinha. Informações historiográficas afirma que Ipanema operou predominantemente com redução indireta e Sardinha exclusivamente com redução direta como as três amostras do Sítio de Ipanema apresentaram altos teores de $\mathrm{FeO}$, é possível estabelecer uma relação entre o processo produtivo e o teor de $\mathrm{FeO}$ das inclusões de escória.

A diferença de teor de $\mathrm{FeO}$ pode ser creditado a detalhes do processo de forjamento, tanto da redução direta quanto do refino do gusa. Durante o forjamento, diversos materiais eram introduzidos para baixar o ponto de fusão da escória ou da ganga presente na superfície ou mesmo no interior da massa de ferro. Não se dispõe de muita informação sobre isso na literatura. Eschwege [6] afirma conhecer

* Contribuição técnica ao $69^{\circ}$ Congresso Anual da ABM - Internacional e ao 14ํㅡㄹ ENEMET - Encontro Nacional de Estudantes de Engenharia Metalúrgica, de Materiais e de Minas, 21 a 25 de julho de 2014, São Paulo, SP, Brasil. 


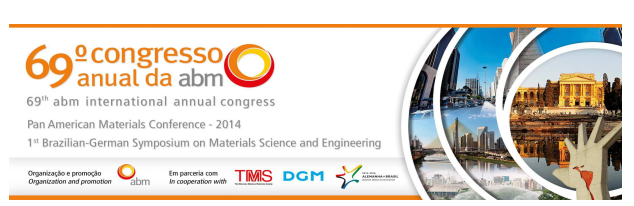

doze procedimentos diferentes de refino, e que cada minério exigia adaptações particulares do processo de refino. Escória do alto-forno era constituinte comum da escória de descarbonetação do gusa e também era comum jogar areia na superfície da barra que saía do forno, pois $\mathrm{SiO}_{2}$ baixa o ponto de fusão do $\mathrm{FeO}$.

\section{CONCLUSÃO}

O uso das técnicas de microanálise EDS juntamente com tratamentos estatísticos dos dados mostrou-se útil para discutir as questões de proveniência das peças de IAraçoiaba.

1. A hipótese de que o intersepto passa pela origem não foi aplicável nestas análises, já que o valor do intersepto é representativo, para maioria dos casos.

2. Não foi possível obter uma assinatura química das peças de Ipanema com base nas relações $\% \mathrm{SiO}_{2}$ versus $\% \mathrm{Al}_{2} \mathrm{O}_{3}, \% \mathrm{FeO}$ versus $\% \mathrm{SiO}_{2}$ e $\% \mathrm{Al}_{2} \mathrm{O}_{3}$ versus $\% \mathrm{MgO}$.

3. As amostras do Sítio de Sardinha, produzidas por processo de redução e forjamento, possuem menor teor de FeO.As análises de conjunto de peças podem ocasionar em um falso resultado, já que as amostras podem ter origens diferentes apesar de estarem no mesmo sitio arqueológico. Os resultados mostram que é preferível usar as análises individuais das peças.

\section{Agradecimentos}

Indicar pessoas que colaboraram na execução da pesquisa ou que proporcionaram auxílio técnico. Quando houver Auxílios ou Bolsas provenientes de órgãos de fomento, os autores deverão fazer referência ao nome da instituição financiadora da pesquisa, ao ano e ao número do processo a que se refere o apoio.

\section{Agradecimentos}

Os autores gostariam de agradecer a:

- Ao Ministério do Meio Ambiente - MMA, Instituto Brasileiro do Meio Ambiente e dos Recursos Naturais Renováveis - IBAMA, Instituto Chico Mendes de Conservação da Biodiversidade - ICMBio, pela autorização de retirada das peças para análises;

- Ao Dr. Luciano Bonatti Regalado e a Arquéologa Dra Margarida Andreatta pelas amostras cedidas e apoio;

\section{REFERÊNCIAS}

1 Dillmann $\mathrm{P}$, L'Héritier $\mathrm{M}$, Slag inclusion analyses for studying ferrous alloys employed in French medieval buildings: supply of materials and diffusion of smelting processes. Journal of Archaeological Science, 2007: 34(11): 1810-1823.

2 Landgraf FJG, Tschiptschin AP, Goldenstein H. Notas sobre a história da metalurgia no Brasil (1500-1850), 2008. http://www.pmt.usp.br/notas/notas.htm. Acessado em 16/03/2014

3 Landgraf FJG, Araujo PEM, et al. (2010). Start up da siderurgia brasileira.

4 Zequini A. (2006). Arqueologia de uma Fábrica de Ferro: morro de Araçoiaba, séculos XVI-XVIII. Museu de Arqueologia e Etnologia de São Paulo, MAE-USP. São Paulo, Universidade de São Paulo. Brasil.

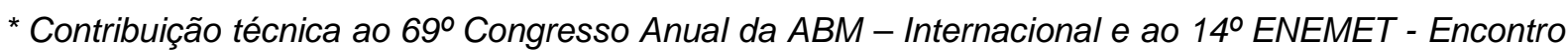
Nacional de Estudantes de Engenharia Metalúrgica, de Materiais e de Minas, 21 a 25 de julho de 2014, São Paulo, SP, Brasil.
} 
5 Azevedo CRF, Landgraf FJG, Maia RR. Análise Microestrutural de Objetos da Real Fábrica de Ferro São João do Ipanema. Revista Matéria, Rio de Janeiro, Editora Scielo. Data de submissão: [24/05/2012].

6 Eschwege GB. Memória sobre as dificuldades das Fundições, e Refinações nas Fábricas de Ferro, para ganhar este metal na maior quantidade, e da melhor qualidade para os diferentes fins. Memórias Econômicas da Academia Real de Ciências de Lisboa, Tomo IV, Lisboa, 1812.

7 Blakelock E, Torres MM, Veldhuijzen HA, Young T. Slag inclusions in iron objects and the quest for provenance: an experiment and a case study, Journal of Archaeological Science, 2009; 36: 1745-1757.

8 Buchwald VF. Slag Analysis as a Method for the Characterizatio and Provenancing of Ancient Iron Objects. Journal of Archaeological Science

9 Pagès G, et al., A study of the Roman iron bars of Saintes-Maries-de-la-Mer (Bouchesdu-Rhône, France). A proposal for a comprehensive metallographic approach. Journal of Archaeological Science, 2011; 38(6): 1234-1252

10 Starley D. Determining the Technological Origins of Iron and Steel. Journal of Archaeological Science, 1999; 26(8): 1127-1133

11 Desaultya A.-MMC, Dillmann P, Jorona JL, Fluzinb P.. Vol, A provenance study of iron archaeological artefacts by Inductively Coupled Plasma-Mass Spectrometry multielemental analysis. 2008; 63.

12 Mason RLGR, Hess JL. Statistical Design and Analysis of Experiments, ed. S. Edition. 2003, New Jersey EUA: John Wiley \& Sons, Inc.

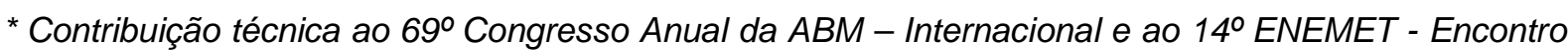
Nacional de Estudantes de Engenharia Metalúrgica, de Materiais e de Minas, 21 a 25 de julho de 2014, São Paulo, SP, Brasil. 